\title{
Involuntary movement in an emotionally labile girl : Think of Wilson's Disease
}

\author{
Chowdhury Rifat Niger ${ }^{1}$, Raj Chowdhury², Chowdhury Akram-Uz-Zaman ${ }^{3}$, Tamzeed Hossain ${ }^{4}$, Rawshan Arra \\ Khanam $^{5}$
}

\begin{abstract}
Wilson's Disease (WD) is a rare, autosomal recessive, inborn error of copper metabolism, which is caused by a mutation in the copper-transporting gene, ATP7B. The presentation is usually neurologic or hepatic or both, which is seen in $40 \%$ of the patients. The diagnosis depends primarily on the clinical features, the biochemical parameters and the presence of the Kayser - Fleischer ring. Here, we are reporting a 13 years old girl who was affected by Wilson's disease, with both neurological manifestations \& hepatic involvement.
\end{abstract}

Key-words : ATP7B, Dystonia, Kayser-Fleischer ring (K-F ring), Wilson's Disease (WD).

\section{Introduction :}

Wilson's Disease (WD), which is also known as hepatolenticular degeneration, was first defined by Dr Samuel Alexander Kinnier Wilson in 1912. ${ }^{1}$ a Wilson's disease (WD) is a rare, autosomal recessive disorder of the copper metabolism, resulting from mutation in the copper-transporting gene, ATP7B. The WD gene, ATP7B, is located on chromosome 13q14.3, which encodes a metal-transporting P-type adenosine triphosphatase (ATPase), which is expressed mainly in hepatocytes. The incidence of WD is estimated to be 1 in 30,000 individuals and the carrier frequency is approximately 1 in $90 .^{2}$ An absent or a reduced function of the ATP7B protein leads to a decreased hepatocellular excretion of copper into bile. This results in hepatic copper accumulation and cellular injury. Eventually, copper is released into the bloodstream and it is deposited in other organs, notably in the brain, kidneys and the cornea. The hepatic production and the secretion of the ceruloplasmin protein without copper and apoceruloplasmin, result in the decreased blood level of ceruloplasmin which is found in most of the patients with $\mathrm{WD}$, due to the reduced half-life of apoceruloplasmin ${ }^{3}$. The manifestations are more likely to be hepatic in early childhood and to be neurological in adolescents.

1. Specialist, Dept of Gastroenterology, United Hospital Limited, Gulsan-2

2. Resident Medical Officer, Dept. of Gastroenterology, BIRDEM General Hospital.

3. General Practitionar (GP), Sydney, Australia

4. Senior House Officer, Dept. of Gastroenterology, United Hospital Limited

5. Junior Consultant, Dept. of Pulmonology, United Hospital Limited

\section{Corresponding Author:}

Dr. Chowdhury Rifat Niger

MBBS, MRCP (UK)

Specialist, Dept of Gastroenterology,

United Hospital Limited, Gulsan-2

Email: doct.rifat@yahoo.com

Contact number: +88 01819-486113
The neurological manifestations of Wilson's disease can vary extremely and they are often diagnosed after long delays. ${ }^{4}$ Here, we are reporting Wilson's disease with neurological \& hepatic presentations in a 13 years girl.

\section{Case Report:}

13 years old Bangladeshi girl presented to us with $\mathrm{C} / \mathrm{O}$ involuntary movement of neck, hands \& legs in the form of repetitive purposeless abnormal movements associated with abnormal psychological behavior like restlessness, agitation $\&$ emotional variation like sudden smiling or crying for last 3 years. She also complained of having wrist, elbow \& knee joint pain for last 2 years.

She gradually developed abnormal posturing of neck, hand, leg \& finally became unable to move around. She had progressive dysphagia associated with slurring of speech \& became more restless for last $1 \frac{1}{2}$ years. She complained of inability to walk \& recurrent fall for last 1 year. She developed yellowish discoloration of eye \& urine for last 2 months.

She was delivered by normal \& uneventful vaginal delivery. She is the youngest daughter of non-consangunious parents. Upto 10 years of age she had normal developmental milestones. Then she gradually developed above mentioned problems for last 3 years. She has 4 brothers. One of her brother also has similar symptoms. On admission : She was conscious, oriented, icteric \& anaemic. She has hepatomegaly, liver was palpable about $3 \mathrm{~cm}$ from right subcostal margin in mid-clavicular line. It was soft \& non-tender with smooth surface. Cervical dystonia with extensor deformity of neck, flexor deformity of elbow, wrist, knee joints were present. Increased muscle tone \& exaggerated deep tendon reflexes of both upper \& lower limbs with restriction of movement, ataxia \& dysarthria were also present.

Laboratory workup reveled :24 hours urinary $\mathrm{Cu}: 110 \mathrm{ug} / 1$ (Normal Range :upto :50 ug/l), S.Ceruloplasmin: $24 \mathrm{mg} / \mathrm{dl}$ (Normal Range :20-60 mg/dl ),S.Bilirubin: $2.9 \mathrm{mg} / \mathrm{dl}$ ( Normal upto $<1 \mathrm{mg} / \mathrm{dl}$ ), USG of whole abdomen : liver is $14 \mathrm{~cm}$ with normal echotexture. Slit Lamp examination revealed KF ring 
present in both eyes.Other lab test like Full blood count, S.Electrolytes, renal function tests were within normal range. Liver biopsy was not done because her parents did not give consent for invasive procedure. Genetic mutation test could not be done due to financial constraints.

The T2(MRI) - weighted axial sequence through pons revealed the "face of the miniature panda", which was also suggestive of Wilson's disease (Fig 1). The diagnosis of Wilson's disease was made on the basis of neurological manifestation like dystonia, ataxia, dysarthia, psychological behaviour, hepatic involvement \& joint pain with the presence of the $\mathrm{K}-\mathrm{F}$ ring in the both eyes, the low serum ceruloplasmin and the 24 hours urinary copper excretion.

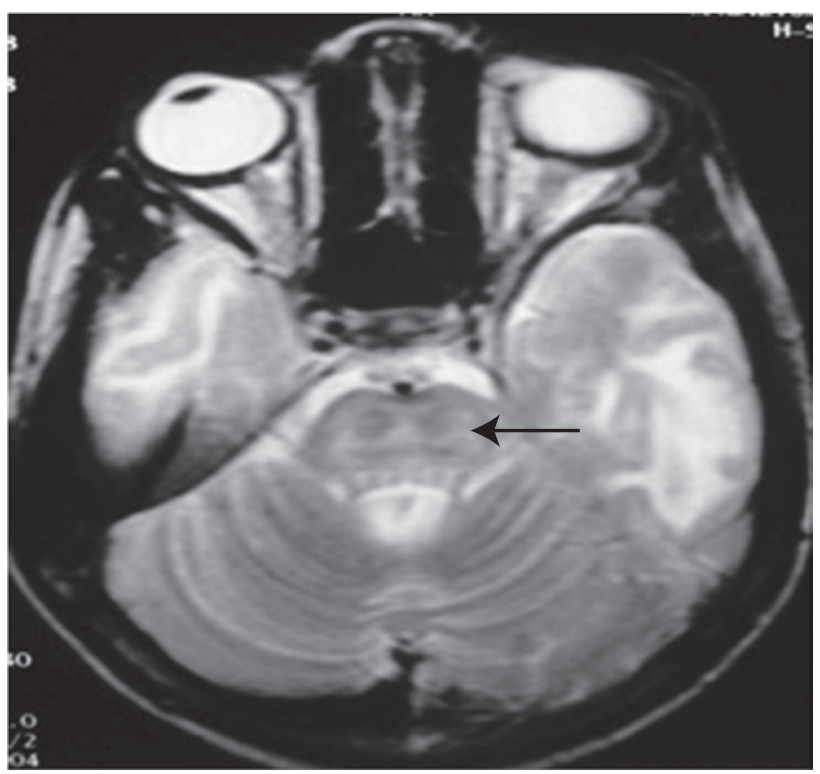

Fig 1 : face of miniature panda (arrow)

The patient was placed on oral zinc (as zinc acetate) at a dose of $1 \mathrm{mg} / \mathrm{kg} / \mathrm{dose}$ of elemental zinc 8 hourly and Trihexiphenidyl to control the dystonia. Oral haloperidol \& perkinil for neuropsychiatric symptoms. She was advised to avoid food with a high copper content, such as chocolates, nuts, legumes, mushrooms, shellfish and liver. Her parents were also advised to avoid the use of copper utensils in the household for the storage of water and for cooking food.

After one week of treatment, we noticed improvement of symptoms of the patient. Dystonia reduced in intensity \& psychiatric symptoms were improved. After two weeks, her joints become more mobile, involuntary movement reduced $\&$ dysphagia \& jaundice improved but hepatomegaly was persistent. We advised her to continue treatment \& come for further follow up after 2 weeks.

We also advised her to come to us with her sibling having similar manifestation for evaluation.

\section{Discussion :}

Wilson's Disease (WD) is a rare, autosomal recessive disease caused by a mutation in the copper-transporting gene, ATP7B. Copper first accumulates in the liver; after the liver storage capacity for copper gets saturated, copper gets redistributed, with accumulation in the nervous system, the cornea, the kidneys and other organs ${ }^{5}$. Most of the patients present in the second decade of life with a primary hepatic presentation, remainder of the patients usually present during the third and fourth decades, with a primarily neurologic or a psychiatric manifestation ${ }^{6}$. However, our case presented with neurological manifestations in the second decade of life (adolescent).The disease was found to manifest at a younger age in Indian children. This could be most likely due to higher average intake of copper in India, which ranges from 5.7 - 7.1 $\mathrm{mg} /$ day and it is higher than the reported $0.34-1.1 \mathrm{mg} /$ day in the western countries. The practice of cooking food and storing drinking water in copper/copper alloy pots might be contributory. 7 .

In Wilson's disease with neurological presentations, the symptoms are predominantly extra pyramidal, like dystonia, tremors, dysphasia, dysarthria, and ataxia. The neurological symptoms are usually secondary to the cerebral copper deposition, which is sufficient to destroy the nerve cells. Our case had neurological manifestations, predominantly dystonia, dysarthria and some cognitive impairment. The serum ceruloplasmin level should not be considered for making a definitive diagnosis, because they are normal in up to $10 \%$ of the affected patients and are reduced in $20 \%$ of the carriers. The Kayser - Fleischer rings can only be diagnosed definitively by an ophthalmologist by using a slit lamp. Urine copper is an important diagnostic tool but it must be collected carefully to avoid contamination. An estimation of the 24hour urinary copper excretion is another reliable test which can be done for the confirmation of WD. The normal excretion of copper is between 20 and $50 \mu \mathrm{g}$ per day.In cases of $\mathrm{WD}$, excretion is increased to in excess of $100 \mu \mathrm{g}$ per day. The "Gold standard" for the diagnosis remains a liver biopsy with quantitative copper assays.

The affected people have values of $>200 \mu \mathrm{g} / \mathrm{gm}$ dry weight of the liver. The copper stains are not reliable ${ }^{8}$. In our case, we had made the diagnosis on the basis of the clinical presentations, the serum ceruloplasmin levels, the 24 hours urinary copper excretion and neuroimaging (MRI). Although a liver biopsy with quantitative copper assay is the "Gold standard", due to the parents' refusal, liver biopsy was not done in our patient. MRI is a very sensitive method for revealing the abnormalities in WD. On the T1 - weighted sequence, hypointensities in the basal ganglia are seen in two-thirds of the cases while in the T2 - weighted images, hyperintensities in the basal ganglia, the white matter, the thalamus or the brainstem are seen. These abnormalities are caused by a neuronal loss, gliosis, degeneration of the fibres, and vacuolisation, which are associated with the increased water content in the brain. The typical 'face of the giant panda' can be seen in the midbrain on the T2 - weighted axial MRI sequence of the brain and the 'face of the miniature panda'(Figure-1) can be seen in the tegmentum region of the pons in the T2 -weighted sequence ${ }^{9}$ as seen in our case.

Penicillamine was considered previously the primary anticopper treatment, but now-a-days, it plays only a minor 
role because of its toxicity and even it often worsens the existing neurologic disease if it is used as the initial therapy. If penicillamine is given, it should always be accompanied by $25 \mathrm{mg} / \mathrm{d}$ of pyridoxine. Trientine is a less toxic chelator and it supplants penicillamine when a chelator is indicated. The drug of choice for Wilson's disease with neurologic/ neuropsychiatric symptoms is Ammonium tetrathiomolybdate, but in most of the countries, it is not available commercially and it is still an experimental medicine. For the patients with hepatitis or cirrhosis, without an evidence of a hepatic decompensation or neurologic/psychiatric symptoms, zinc is the therapy of choice, although some advocate the therapy with trientine. Zinc has proven its efficacy in Wilson's disease and it is essentially nontoxic. It produces a negative copper balance by blocking the intestinal absorption of copper, and it induces hepatic metallothionein synthesis, which sequesters the additional toxic copper. All the presymptomatic patients should be treated prophylactically, since the disease is close to $100 \%$ penetrant ${ }^{10,11}$. Our case was treated with oral zinc (as zinc acetate) in high doses $(1 \mathrm{mg} / \mathrm{kg} /$ dose 8 hourly) and trihexyphenidyl $4 \mathrm{mg} 8$ hourly. After 2 weeks of treatment, an improvement in the symptomatology was seen. Genetic therapy and hepatocyte transplantation represent the future curative treatments for WD, along with the currently available liver transplantation ${ }^{12}$.

\section{Conclusion}

Wilson's disease is an uncommon autosomal recessive, metabolic disorder, which is often missed but easily treatable. A high index of suspicion is required while dealing with adolescents and young adults with abnormal movements and neuro-psychiatric abnormalities. A high degree of suspicion and an early detection of WD is difficult but an early initiation of the treatment can prevent a catastrophic outcome.

\section{References:}

1. Wilson SAK. Progressive lenticular degeneration: a familial nervous disease associated with cirrhosis of the liver. Brain. 1912;34: 295-509.

2. Huster D. Wilson's disease. Best Pract Res Cl Ga. 2010; 24(5): 531-39.

3. Holtzman NA, Gaumnitz BM. Studies on the rate of release and turnover of ceruloplasmin and apoceruloplasmin in rat plasma. J Biol Chem. 1970; 245: 2354-58.

4. Kalra V. Wilson's disease: early onset and lessons from a pediatric cohort in India. Indian Pediatr. 2000;37: 595-601.

5. Sokol RJ. Wilson's Disease and Indian childhood cirrhosis. In: Diseases in Children. Eds. Suchy, Fredrick J. St Louis, Mosby Year Books Inc, 1994; 747-72.

6. Walshe JM. The Liver in Wilson's Disease (Hepatolenticular Degeneration) In: Diseases of the Liver. Eds Schiff L, Schiff ER, Philadelphia, J. B. Lippincott. 1982; 1037-84.

7. Ferenci P, Czlonkowska A, Stremmel W, Houwen R, Rosenberg W,Schilsky M. et al EASL clinical practice guidelines: Wilson's disease. J Hepatol. 2012; 56(3):671-85.

8. George J. Brewer. Wilson Disease. Harrison's Principle of Internal Medicine.17th Edn, Mc Graw Hill Medical. 2008; 2449-51.

9. Page RA. Clinical correlation of brain MRI and MRS abnormalities in patients with Wilson disease. Neurology. 2004; 63: 638-43.

10. Kuruvilla A, Joseph S. 'Face of the giant Panda' sign in Wilson's disease: revisited. Neurol India. 2000; 48: 395-96.

11. Wu ZY, Lin MT, Murong SX, Wang N. Molecular diagnosis and prophylactic therapy for presymptomatic Chinese patients with Wilson disease. Arch Neurol-Chicago. 2003; 60(5):737-41.

12. Malhi H, Joseph B, Schilsky ML, Gupta S. Development of cell therapy strategies to overcome copper toxicity in the LEC rat model of Wilson disease. Regen Med. 2008; 3(2):165-73. 